\title{
CALIBRATION OF COSMO-SKYMED SAR DATA FOR HYDROLOGY APPLICATIONS
}

\author{
Daniele Riccio $^{1}$, Gerardo Di Martino ${ }^{1,2}$, Antonio Iodice ${ }^{1}$, Youssouf Koussoube ${ }^{3}$, Alfonso Davide Pinelli ${ }^{2}$, \\ Giuseppe Ruello ${ }^{1,2}$ \\ ${ }^{1}$ University of Napoli "Federico II", \\ Via Claudio 21, 80125, Napoli, Italy \\ ${ }^{2}$ Engineering Without Borders - Napoli, Italy \\ ${ }^{3}$ Departement de Geologie, UFR/SVT, \\ University of Ouagadougou, Burkina Faso
}

\begin{abstract}
In this paper we present a procedure for the calibration of SAR data devoted to extract physical information from Cosmo-Skymed images. The main purpose is the extraction of land cover indexes for hydrology applications. The calibration procedure was implemented in a rural context in Burkina Faso, where along with the technical challenges we faced socio-economic problems. A set of handmade trihedral corner reflectors were deployed and their response measured from SAR images. The results were used for calibration of an erosion product.
\end{abstract}

Index Terms - Synthetic Aperture Radar, calibration, trihedral corner reflector

\section{INTRODUCTION}

The Sahel region is characterized by a semi-arid climate, with the alternation of rainy (from June to October) and dry (From November to May) seasons. Almost $80 \%$ of the population relies on agriculture. During the dry season the scarcity of water hampers the field cultivation; during the wet season, the precipitations often cause floods and they bring damages to the local agriculture and the people living condition. For these and other structural reasons, it is estimated that in the Sub-Saharan Africa almost $26 \%$ of the people is undernourished [1]. The identification, monitoring and management of the water resource is crucial for creating better conditions for the local people. The use of remote sensing in this area could be a significant support for people in charge of taking decisions.

Despite these needs, there are still technical and socioeconomical factors that limit the diffusion of remote sensing techniques for hydrology in semi-arid regions. In this paper we present a project devoted to use high resolution satellite data for calibrating hydrologic models in semi-arid regions at no cost for local people. A case study was developed in Burkina Faso. Along with the technical characteristics, the keys for the project success have been related with the local people participation.

The employed data were provided at no cost by the Italian Space Agency, in the frame of the Announcement of Opportunities for the exploitation of high resolution X-band Cosmo SkyMed data. The project is based on the fusion of remote sensing and hydrologic models and data. The SAR data were used for calibrating the hydro-geological models, providing information on soil erosion, floods, land cover, soil moisture and so on. The extraction of this and more information often requires a calibration procedure that calls for the installation of corner reflectors [2]. Therefore, we developed a procedure for the calibration of the radar images by means of trihedral corner reflectors. In the next section we present the methodology employed for building the corners with the participation of local researchers and manufacturers. Three trihedral corner reflectors with side of $75 \mathrm{~cm}(25 \lambda$ at X-band) were built by local workers by using recycled materials. The positions were decided in accordance with the local farmers in the area of Bidi, a small village located $40 \mathrm{~km}$ north of Ouahigouya, the capital of the Yatenga region, in the north of the country. The integral method ([3]-[4]) is employed for the image calibration processing. In Section 2 we present the rationale that governed the corner reflector building and positioning. Section 3 recalls the basic theory on SAR signal external calibration. In Section 4 we present the application of the calibration method for the generation of value added products from a set of SAR images acquired between the 
beginning of the rainy season 2010 and the end of the rain season 2011 in Burkina Faso.

\section{CORNER REFLECTOR BUILDING AND POSITIONINIG}

In this section we present the design and implementation of the trihedral corner reflectors used to calibrate the X-band high resolution SAR images acquired by the Italian sensor Cosmo SkyMed. We built trihedral corner reflectors, with a side of $75 \mathrm{~cm}$, that, at X-band, corresponds to 25 wavelengths and it is comparable with the Cosmo-SkyMed resolution cell $(1 \mathrm{~m}$ and $3 \mathrm{~m}$ for Spotlight and Stripmap operational modes, respectively). This choice is a trade-off between the manufacturing limits, the portability of the object, its interaction with the terrain and the significance of the expected signal to noise ratio. Theoretical simulations of the corner radar cross section show that the chosen values allow angle stability coherent with the precision that we expect to reach with the available positioning instruments. The theoretical peak radar cross section $\sigma_{t}$ of the built reflectors is:

$$
\sigma_{t}=\frac{4 \pi a^{2}}{3 \lambda^{2}} .
$$

The corners were built by local manufacturers, with recycled materials. Such a choice has two consequences:

1. no waste was produced in the country;

2. the local people participated to all the steps of the building procedure.

In Figure 1 significant moments of the corner reflector construction are shown, along with a snapshot of produced calibrator.

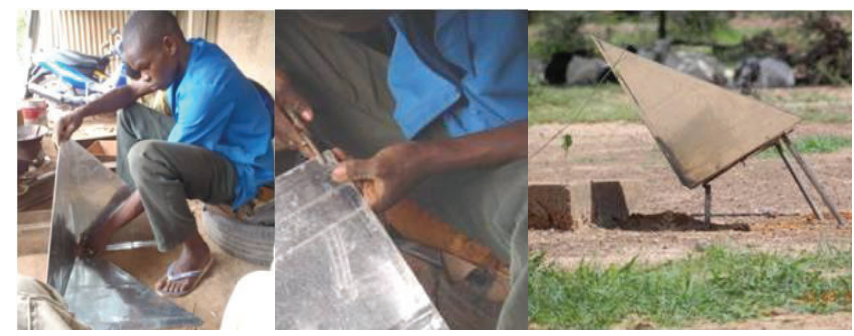

Figure 1: Sequence of the corner manufacturing and snapshot of the built object.

The use of rudimental instruments and recycled materials poses problems of reliability. In order to reduce the noise produced by the building procedure, we deployed three corner reflectors in a limited area around the Bidi village. The calibrators were placed in eroded areas, where the soil does not absorb rain so that it presents an almost invariant radar cross section. In Figure 2 we show the area of study, as seen by Google Earth, and a zoom on the locations of two of the deployed reflectors.
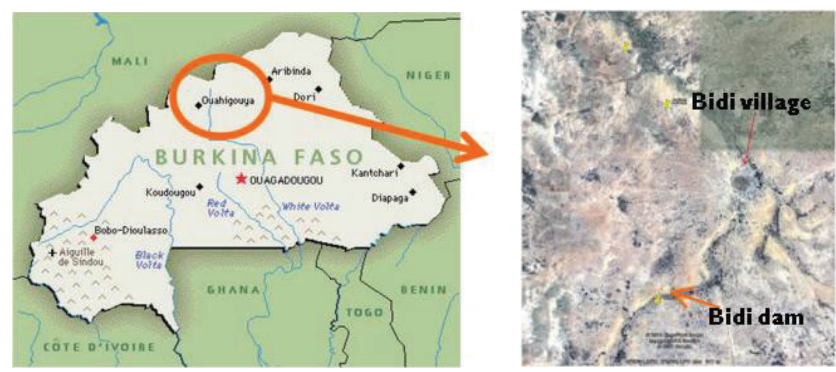

Figure 2: The case study was developed close to the Bidi village, in the North of Burkina Faso.

The corner reflectors were placed on August 22, 2011; since then, 1 Cosmo-Skymed Stripmap image per month was acquired until December 2011. In Figure 3 and Figure 4 we present a comparison between a Google Earth and a CosmoSkymed Spotlight (1 $\mathrm{m}$ of spatial resolution) view of two areas of almost $750 \times 600 \mathrm{~m}^{2}$ close to the Bidi village and the Bidi dam, respectively. In addition, for each area we present a range cut relevant to the deployed corner reflectors.

a)

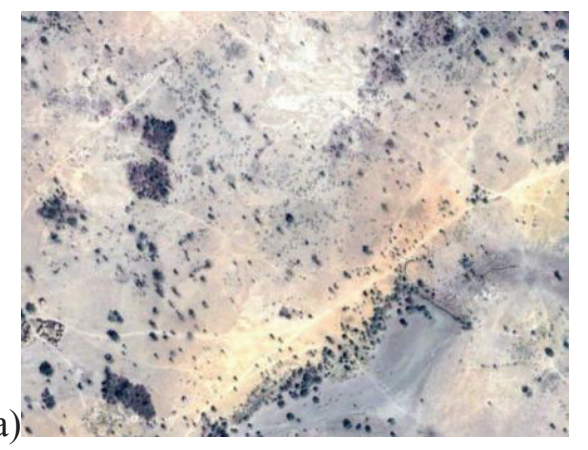

c)
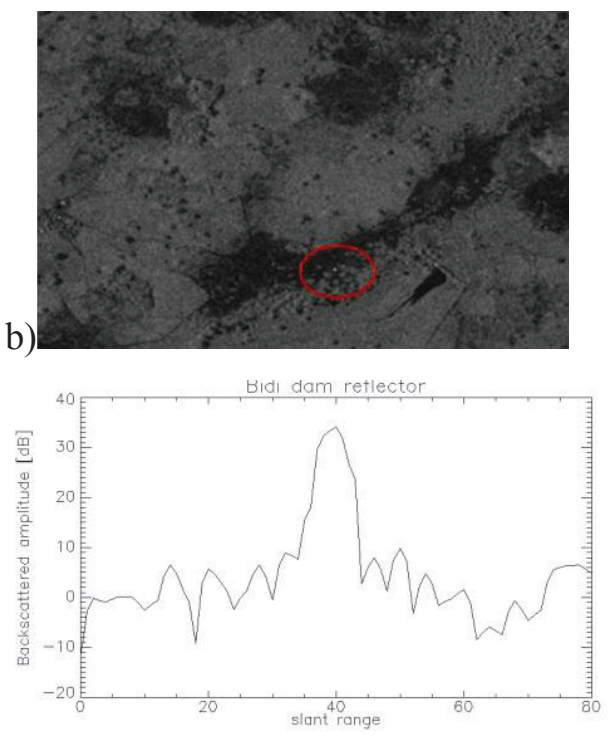

Figure 3: Google Earth view (a), SAR image (b) and range cut (c) relevant to the Bidi dam area. 


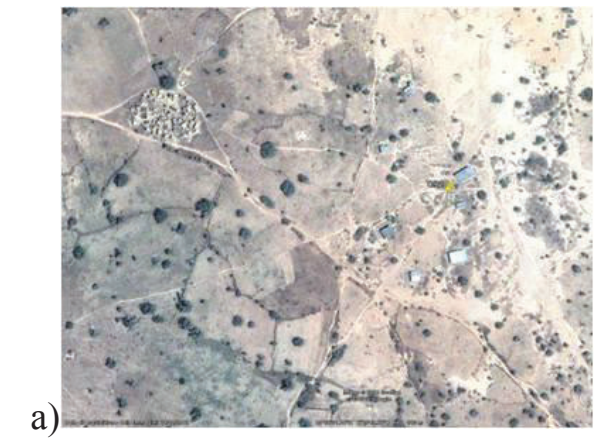

b)

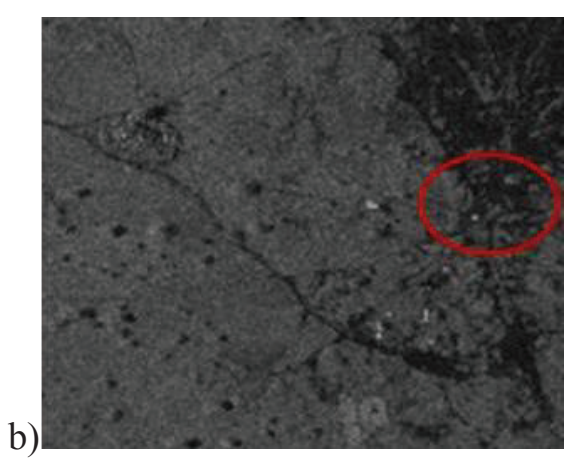

c)

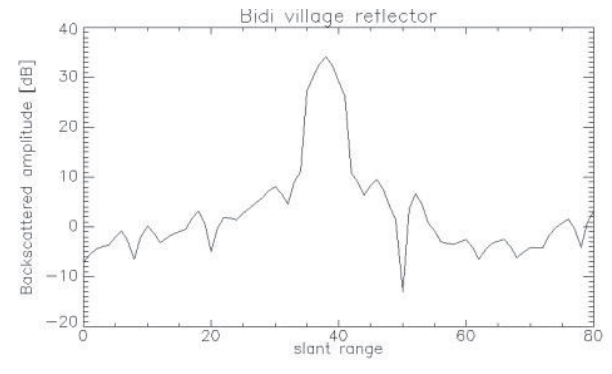

Figure 4: Google Earth view (a), SAR image (b) and range cut (c) relevant to the Bidi village area.

The corner reflectors provided comparable responses (see the range cuts of Fiures $3 \mathrm{c}$ and $4 \mathrm{c}$ )), which are in accordance with the expected performances. The absolute image calibration can be performed with peak and integral methods. In addition, corner reflectors can be used for relative calibration for images acquired in different dates. The peak method is more sensitive to phase or manufacturing errors and to an accurate procedure of sinc function shape reconstruction. The integral method provides better results thanks to its robustness, which is a crucial characteristic in the presented context. In the following, we recall some basic concepts of the SAR calibration methods and, in Section 4, we present a practical use of a relative calibration procedure for multi-time analysis of SAR images.

\section{CALIBRATION METHODS}

A calibration procedure allows relating the SAR image pixel values to the relative radar cross sections. In general, the image power $P$ after standard SAR processing can be expressed as [2]:

$$
P=k_{s} \sigma+k_{n} n,
$$

where $k_{s}$ and $k_{s}$ are the radar gains in presence of signal and noise [2]; $\sigma$ and $n$ are the radar cross section of the observed area and of the noise, respectively. In the frame of the integral approach [4], the calibration factors are evaluated by integrating the image power over an area containing the power scattered by the corner reflector $P_{p}$, and subtracting off the power corresponding to a homogeneous scatterer $P_{u}$ and the receiver noise power $P_{n}$.

The function $P_{p}$ is calculated from integration over an area $A_{p}$ large enough to contain all the energy of the impulse response. The power $P_{u}$ is the response over a homogeneous area $A_{u}$ with the same characteristics of the terrain where the corner is located.

If the signals from the homogeneous clutter, the noise and receiver noise are mutually uncorrelated, the backscattering coefficient may be expressed as:

$$
\sigma^{0}=\frac{\left(\sigma / A_{n u}\right)\left[P_{n u}-P_{n}\left(A_{n u} / A_{n}\right)\right]}{P_{p n u}-P_{n u}\left(A_{p n u} / A_{n u}\right)},
$$

where $\sigma$ is the known radar cross section of the reflector, $A_{n u}$ is the ground area containing receiver noise and the clutter signal, $A_{p n u}$ is the area containing the reflector, the receiver noise and uniform clutter signal. The integrated powers over these three specified areas are $P_{n u}$ and $P_{p n u}$, respectively.

In the frame of the peak method, the shape and peak value of a point reflector response depend on the form of the weighting functions used to reduce side-lobes. The normalized radar cross section can be derived as [4]:

$$
\sigma^{0}=\frac{\sigma \sin \theta}{\rho_{r} \rho_{a} C_{p}},
$$

where $C_{p}$ is the ratio of the corrected for noise and clutter peak reflector estimate to the adjacent average noise corrected clutter, $\rho_{a}, \rho_{r}$ are the azimuth and slant range resolutions, respectively [4].

Integral method is more robust with respect to image defocusing and it has often preferred with respect to the peak method [5] especially when imperfect targets as handmade corner reflectors were used.

In many applications, a relative calibration is sufficient for change detection or analysis of a series of data. The calibration factors can be evaluated by ratioing the pixel intensities on point-target whose theoretical response is known. 


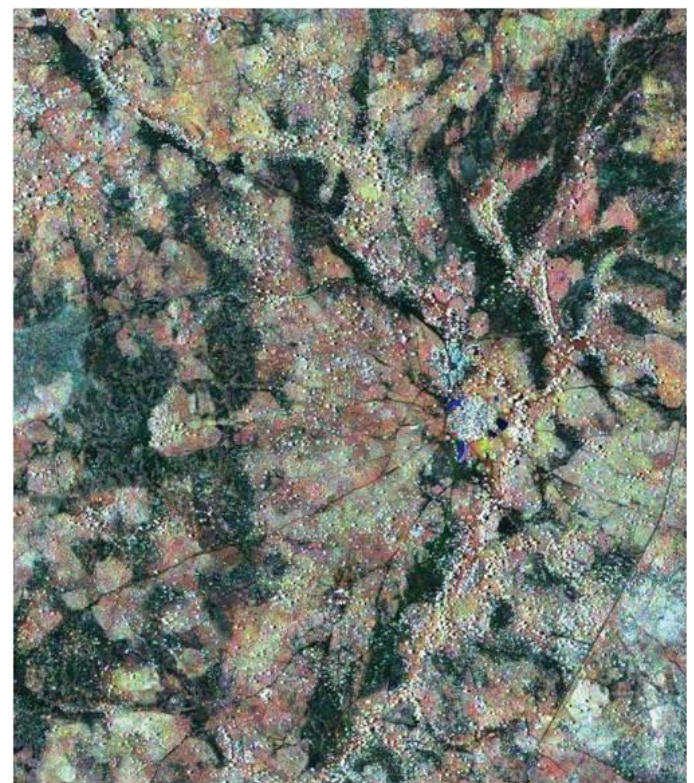

Figure 5: RGB color image of the Yatenga region, Burkina Faso.

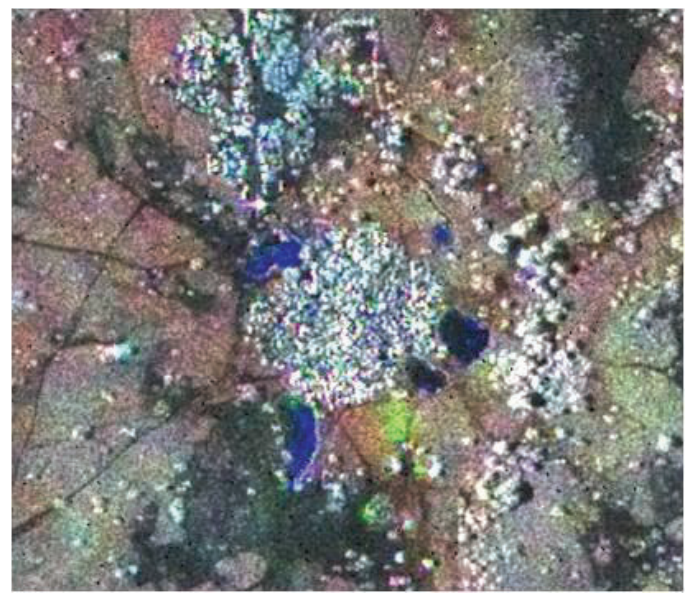

Figure 6: Detail of the RGB color image of the Yatenga region, Burkina Faso.

\section{HYDROLOGY APPLICATIONS}

In this Section, we present how the built corner reflectors were used for the classification of land cover in a rural area, by the combination of intensity SAR images. This study was conducted for extracting relevant hydrologic features from multi-temporal SAR images. We show here a classification algorithm based on the ground reflectivity variations, devoted to identify hydrologic characteristics of the fields close to the Bidi village, in the North of Burkina Faso.

The relative calibration guarantees that the ratio between the pixel values is representative of the ratio between the soil reflectivity. The red, green and blue channels were loaded with images acquired in October, November and December, respectively.

The color levels of Figure 5 can be associated to the land use by considering that the observed area is characterized by a wet season that goes from June to October. Since October, the terrains start to lose their vegetation cover, with a decreasing in the soil reflectivity. The process is quicker in areas far from the water sources. Red areas are regions where we expect that the vegetation was lost between October and November. Dark pixels represent the eroded lands that do not absorb the rain and that present a low reflectivity in all the seasons. Note that, close to the village water reservoirs, some green slots represent cultivated field, that was irrigated also in the dry season, allowing counter-season cultivation until November, see Figure 6.

\section{CONCLUSIONS}

The presented work is framed in a project devoted to exploit Cosmo-SkyMed high resolution data for calibration of hydrological models. We proposed an efficient approach for building and deploying corner reflectors in low income countries, and we developed a case study in Burkina Faso. The corner reflectors were built with the involvement of local people. Such an approach guaranteed not only the fulfillment of the required technical specifics, but also the transfer of knowledge to and from local people, that was a key factor for the success of the whole project. A significant example of the use of the corner reflector for the extraction of land cover of a rural area in the North of Burkina Faso was presented.

\section{REFERENCES}

[1] FAO, The State of Food and Agriculture 2010-2011, FAO, Rome, 2011.

[2] A. Freeman, "SAR calibration: an overview", IEEE Trans. Geosci. Remote Sens., vol.30, n.6, pp. 1107-1121, Nov.1992.

[3] L. M. H. Ulander, "Accuracy of Using Point Targets for SAR Calibration”, IEEE Trans. Aerosp. Electron. Syst., vol. 27, no. 1, pp. 139-148, Jan. 1991.

[4] A.L. Gray, P.W. Vachon, C.E. Livingstone and T.I. Lukowski. "Synthetic Aperture Radar calibration using reference reflector", IEEE Trans. Geosci. Remote Sens., vol. 28, no. 3, pp. 373-383, May 1990.

[5] B. J. Döring, P. Looser, M. Jirousek, and M. Schwerdt, "Point Target Correction Coefficients for Absolute SAR Calibration", Proceedings IEEE I2TMC 2011, pp. 1-6, 2011. 